CRYSTALLOGRAPHIC COMMUNICATIONS

ISSN 2056-9890

Received 1 December 2016

Accepted 23 December 2016

Edited by S. Parkin, University of Kentucky, USA

Keywords: crystal structure; reductive ammonolysis; streptozocin; rubidium cation; methyldiazotate anion.

CCDC reference: 1524271

Supporting information: this article has supporting information at journals.iucr.org/e

\section{Crystal structure of rubidium methyldiazotate}

\author{
Tobias Grassl and Nikolaus Korber*
}

Institut für Anorganische Chemie, Universität Regensburg, Universitätsstrasse 31, 93053 Regensburg, Germany.
*Correspondence e-mail: nikolaus.korber@chemie.uni-regensburg.de

The title compound, $\mathrm{Rb}^{+} \cdot \mathrm{H}_{3} \mathrm{CN}_{2} \mathrm{O}^{-}$, has been crystallized in liquid ammonia as a reaction product of the reductive ammonolysis of the natural compound streptozocin. Elemental rubidium was used as reduction agent as it is soluble in liquid ammonia, forming a blue solution. Reductive bond cleavage in biogenic materials under kinetically controlled conditions offers a new approach to gain access to sustainably produced raw materials. The anion is nearly planar [dihedral angle $\mathrm{O}-\mathrm{N}-\mathrm{N}-\mathrm{C}=-0.4(2)^{\circ}$ ]. The $\mathrm{Rb}^{+}$cation has a coordination number of seven, and coordinates to five anions. One anion is bound via both its $\mathrm{N}$ atoms, one by both $\mathrm{O}$ and $\mathrm{N}$, two anions are bound by only their $\mathrm{O}$ atoms, and the last is bound via the $\mathrm{N}$ atom adjacent to the methyl group. The diazotate anions are bridged by cations and do not exhibit any direct contacts with each other. The cations form corrugated layers that propagate in the (101) plane.

\section{Chemical context}

The crystal structure of the title compound was determined in the course of investigations regarding the reactivity of carbohydrates towards alkali metals and $\mathrm{NH}_{3}$ in solutions where liquid ammonia itself is used as solvent. The starting material, streptozocin, was commercially available and used as shipped.

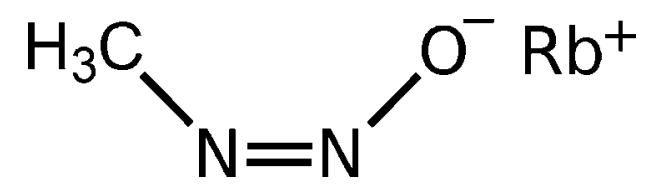

\section{Structural commentary}

The methyldiazotate anion is found to exist in the cis configuration, which is in correspondence with the equivalent potassium species (Müller et al., 1963; Huber et al., 1965). The structure of the diazotate anion has been further discussed by Suhr (1963) and by Kübler \& Lüttke (1963).

The title compound does not contain any solvent molecules, which is unusual for ionic species crystallized from liquid ammonia. The anion is nearly planar, having an $\mathrm{O} 1-\mathrm{N} 1-$ $\mathrm{N} 2-\mathrm{C} 1$ torsion angle of $-0.4(2)^{\circ}$. Five direct anion-cation contacts can be observed, with maximum bond lengths of $d(\mathrm{Rb}-\mathrm{O})=2.9871(12) \AA$ and $d(\mathrm{Rb}-\mathrm{N})=3.1656(15) \AA$. The rubidium cation has a coordination number of seven, in which five anions can be observed in its direct environment (Fig. 1). The coordination to the cation is both side-on and terminal: one anion is bound via both its $\mathrm{N}$ atoms, one by both $\mathrm{O}$ and $\mathrm{N}$, two anions are bound only via $\mathrm{O}$, and the remaining anion is bound via the $\mathrm{N}$ atom adjacent to the methyl group. 


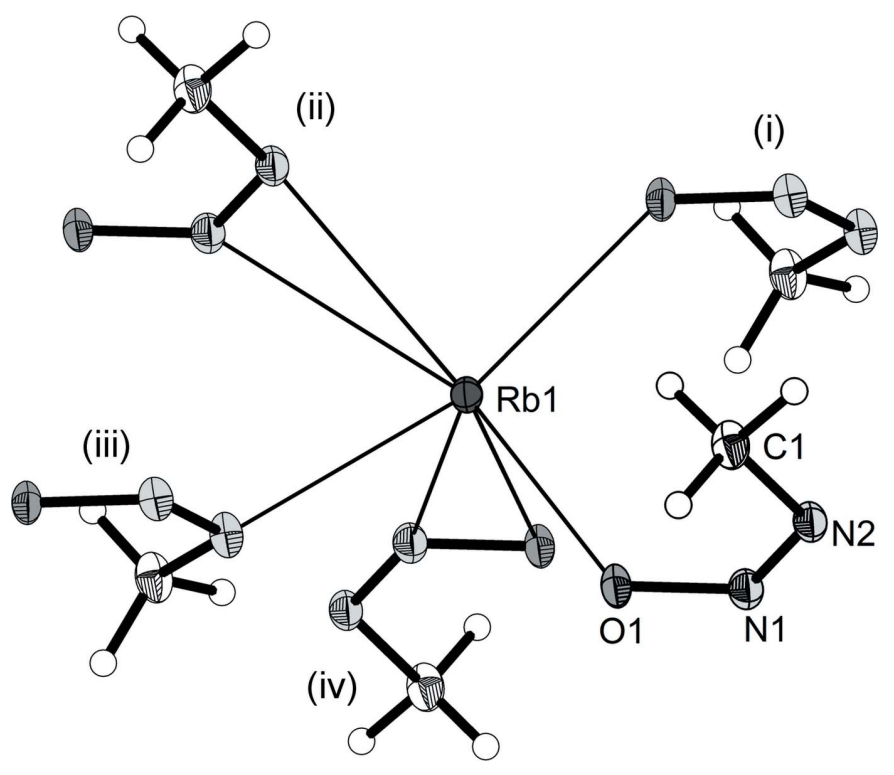

Figure 1

The coordination environment of the $\mathrm{Rb}^{+}$cation. Displacement ellipsoids are drawn at the $50 \%$ probability level. [Symmetry codes: (i) $-\frac{1}{2}+x, \frac{3}{2}-y$, $\frac{1}{2}+z$; (ii) $-1+x, y, z$; (iii) $-\frac{1}{2}+x, \frac{3}{2}-y, \frac{1}{2}+z$; (iv) $1-x, 1-y, 1-z$.]

\section{Supramolecular features}

The diazotate anions are bridged by cations and do not exhibit any direct contacts to each other. The cations are found to form a corrugated-layer like arrangement within the structure, propagating in the (101) plane (Fig. 2). Although the oxygen atom can act as a hydrogen-bridge acceptor, no such interactions can be found in the structure as the $\mathrm{C}-\mathrm{H}$ bonds are not sufficiently polarized. As the compound is of an ionic nature, electrostatic interactions are the dominant driving force towards the arrangement of the ionic species. An aggregation of methyl groups is therefore not observed.

\section{Computational analysis}

To get a more detailed understanding of the bonding situation in the anion, quantum chemical calculations were carried out at the DFT level (B3LYP functional) using def2-TZVP basis sets. To embed the results in a meaningful frame of reference, diazene and methylnitrosamine were used for comparison

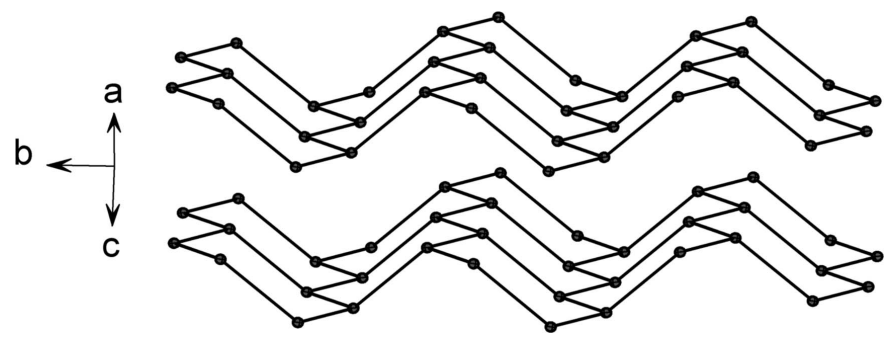

Figure 2

The extended arrangement formed by the cations in the crystal structure. Displacement ellipsoids are drawn at the $50 \%$ probability level.
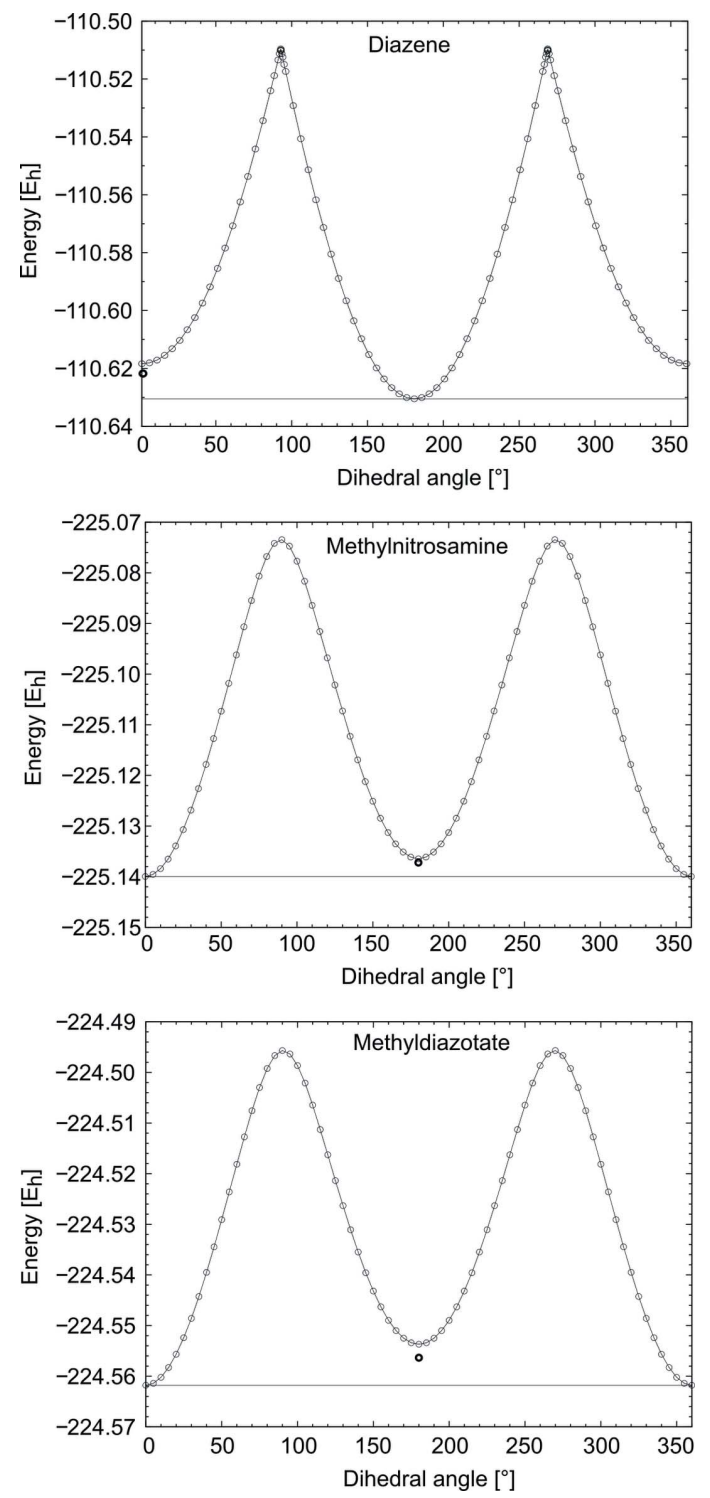

Figure 3

Rotational potentials of diazene, methylnitrosamine and methyldiazotate. The energetic minima were geometrically optimized and are drawn as thick circles.

(Fig. 3). It was found that the methyldiazotate anion tends to have properties most similar to methylnitrosamine. This indicates a high ability to delocalize its $s p^{2}$ electrons.

By analyzing the rotational potential, the energy barrier of the transition between the cis and trans form was determined to be $173.57 \mathrm{~kJ} \mathrm{~mol}^{-1}$. The energetic difference between the two forms is $14.30 \mathrm{~kJ} \mathrm{~mol}^{-1}$, wherein the cis form is energetically preferred. For comparison, the rotational barriers of diazene and methylnitrosamine are calculated to be $317.44 \mathrm{~kJ} \mathrm{~mol}^{-1}$ and $174.58 \mathrm{~kJ} \mathrm{~mol}^{-1}$, respectively. The various computational methods employed have been described by Neese (2012), Weigend \& Ahlrichs (2005), Schäfer et al. (1992, 1994), Eichkorn et al. (1997), Weigend et al. (2003), Metz et al. (2000), Dirac (1929), Slater (1951), Vosko et al. (1980), Becke (1988, 1993), Lee et al. (1988). 


\section{Synthesis and crystallization}

$250 \mathrm{mg}(0.94 \mathrm{mmol})$ of streptozocin and $322 \mathrm{mg}(3.8 \mathrm{mmol})$ of rubidium were placed under an argon atmosphere in a reaction vessel and $20 \mathrm{ml}$ of dry liquid ammonia was condensed. The mixture was stored at $237 \mathrm{~K}$ for two weeks to ensure that all substances were completely dissolved. The flask was then stored at $161 \mathrm{~K}$ for several months. After that period, clear colorless crystals of the title compound could be found at the bottom of the flask.

\section{Refinement}

Crystal data, data collection and structure refinement details are summarized in Table 1. All hydrogen atoms could be located in a difference map and were refined freely.

\section{References}

Agilent (2012). CrysAlis PRO Agilent Technologies Ltd, Yarnton, England.

Becke, A. D. (1988). Phys. Rev. A, 38, 3098-3100.

Becke, A. D. (1993). J. Chem. Phys. 98, 5648-5648.

Bourhis, L. J., Dolomanov, O. V., Gildea, R. J., Howard, J. A. K. \& Puschmann, H. (2015). Acta Cryst. A71, 59-75.

Brandenburg, K. \& Putz, H. (2012). DIAMOND. Crystal Impact, Bonn, Germany.

Clark, R. C. \& Reid, J. S. (1995). Acta Cryst. A51, 887-897.

Dirac, P. A. M. (1929). Proc. R. Soc. London A, 123, 714-733.

Dolomanov, O. V., Bourhis, L. J., Gildea, R. J., Howard, J. A. K. \& Puschmann, H. (2009). J. Appl. Cryst. 42, 339-341.

Eichkorn, K., Weigend, F., Treutler, O. \& Ahlrichs, R. (1997). Theor. Chem. Acc. 97, 119-124.

Huber, R., Langer, R. \& Hoppe, W. (1965). Acta Cryst. 18, 467-473.

Kübler, R. \& Lüttke, W. (1963). Ber. Bunsenges. Phys. Chem. 67, 216.

Lee, C., Yang, W. \& Parr, R. G. (1988). Phys. Rev. B, 37, 786-786.

Metz, B., Stoll, H. \& Dolg, M. (2000). J. Chem. Phys. 113, 2563-2563.

Müller, E., Hoppe, W., Hagenmaier, H., Haiss, H., Huber, R., Rundel, W. \& Suhr, H. (1963). Chem. Ber. 96, 1712-1719.

Neese, F. (2012). WIREs Comput. Mol. Sci. 2, 73-78.

Schäfer, A., Horn, H. \& Ahlrichs, R. (1992). J. Chem. Phys. 97, 25712577.

Schäfer, A., Huber, C. \& Ahlrichs, R. (1994). J. Chem. Phys. 100, 5829-5835.
Table 1

Experimental details.

\begin{tabular}{|c|c|}
\hline \multicolumn{2}{|l|}{ Crystal data } \\
\hline Chemical formula & $\mathrm{Rb}^{+} \cdot \mathrm{CH}_{3} \mathrm{~N}_{2} \mathrm{O}^{-}$ \\
\hline$M_{\mathrm{r}}$ & 144.52 \\
\hline Crystal system, space group & Monoclinic, $P 2_{1} / n$ \\
\hline Temperature (K) & 123 \\
\hline$a, b, c(\AA)$ & $6.8658(1), 8.7614(1), 7.2447(1)$ \\
\hline$\beta\left({ }^{\circ}\right)$ & $114.219(2)$ \\
\hline$V\left(\AA^{3}\right)$ & $397.44(1)$ \\
\hline$Z$ & 4 \\
\hline Radiation type & Mo $K \alpha$ \\
\hline$\mu\left(\mathrm{mm}^{-1}\right)$ & 12.26 \\
\hline Crystal size $(\mathrm{mm})$ & $0.29 \times 0.17 \times 0.15$ \\
\hline \multicolumn{2}{|l|}{ Data collection } \\
\hline Diffractometer & $\begin{array}{l}\text { Agilent SuperNova Dual Source } \\
\text { diffractometer with an Eos } \\
\text { detector }\end{array}$ \\
\hline Absorption correction & $\begin{array}{l}\text { Analytical [CrysAlis PRO } \\
\text { (Agilent, 2012) based on } \\
\text { expressions derived by Clark \& } \\
\text { Reid (1995)] }\end{array}$ \\
\hline$T_{\min }, T_{\max }$ & $0.267,0.267$ \\
\hline $\begin{array}{l}\text { No. of measured, independent and } \\
\text { observed }[I>2 \sigma(I)] \text { reflections }\end{array}$ & $13443,1210,1068$ \\
\hline$R_{\text {int }}$ & 0.051 \\
\hline$(\sin \theta / \lambda)_{\max }\left(\AA^{-1}\right)$ & 0.714 \\
\hline \multicolumn{2}{|l|}{ Refinement } \\
\hline$R\left[F^{2}>2 \sigma\left(F^{2}\right)\right], w R\left(F^{2}\right), S$ & $0.017,0.042,1.04$ \\
\hline No. of reflections & 1210 \\
\hline No. of parameters & 58 \\
\hline $\mathrm{H}$-atom treatment & All H-atom parameters refined \\
\hline$\Delta \rho_{\max }, \Delta \rho_{\min }\left(\mathrm{e} \AA^{-3}\right)$ & $0.56,-0.86$ \\
\hline
\end{tabular}

Computer programs: CrysAlis PRO (Agilent, 2012), olex2.solve (Bourhis et al., 2015), SHELXL2014 (Sheldrick, 2015), DIAMOND (Brandenburg \& Putz, 2012) and OLEX2 (Dolomanov et al., 2009).

Sheldrick, G. M. (2015). Acta Cryst. C71, 3-8.

Slater, J. C. (1951). Phys. Rev. 81, 385-390.

Suhr, H. (1963). Chem. Ber. 96, 1720-1724.

Vosko, S. H., Wilk, L. \& Nusair, M. (1980). Can. J. Phys. 58, 12001211.

Weigend, F. \& Ahlrichs, R. (2005). Phys. Chem. Chem. Phys. 7, 32973305.

Weigend, F., Furche, F. \& Ahlrichs, R. (2003). J. Chem. Phys. 119, 12753-12762. 


\section{supporting information}

Acta Cryst. (2017). E73, 159-161［https://doi.org/10.1107/S2056989016020429]

\section{Crystal structure of rubidium methyldiazotate}

\section{Tobias Grassl and Nikolaus Korber}

\section{Computing details}

Data collection: CrysAlis PRO (Agilent, 2012); cell refinement: CrysAlis PRO (Agilent, 2012); data reduction: CrysAlis PRO (Agilent, 2012); program(s) used to solve structure: olex2.solve (Bourhis et al., 2015); program(s) used to refine structure: SHELXL2014 (Sheldrick, 2015); molecular graphics: DIAMOND (Brandenburg \& Putz, 2012); software used to prepare material for publication: OLEX2 (Dolomanov et al., 2009).

Rubidium methyldiazotate

Crystal data

$\mathrm{Rb}^{+} \cdot \mathrm{CH}_{3} \mathrm{~N}_{2} \mathrm{O}^{-}$

$M_{r}=144.52$

Monoclinic, $P 2_{1} / n$

$a=6.8658(1) \AA$

$b=8.7614(1) \AA$

$c=7.2447(1) \AA$

$\beta=114.219(2)^{\circ}$

$V=397.44(1) \AA^{3}$

$Z=4$

Data collection

Agilent SuperNova Dual Source diffractometer with an Eos detector

Radiation source: SuperNova (Mo) X-ray

Source

Mirror monochromator

Detector resolution: 15.9702 pixels $\mathrm{mm}^{-1}$

phi and $\omega$ scans

Absorption correction: analytical

[CrysAlis PRO (Agilent, 2012) based on

expressions derived by Clark \& Reid (1995)]

Refinement

Refinement on $F^{2}$

Least-squares matrix: full

$R\left[F^{2}>2 \sigma\left(F^{2}\right)\right]=0.017$

$w R\left(F^{2}\right)=0.042$

$S=1.04$

1210 reflections

58 parameters

0 restraints
$F(000)=272$

$D_{\mathrm{x}}=2.415 \mathrm{Mg} \mathrm{m}^{-3}$

Mo $K \alpha$ radiation, $\lambda=0.71073 \AA$

Cell parameters from 8481 reflections

$\theta=3.1-32.1^{\circ}$

$\mu=12.26 \mathrm{~mm}^{-1}$

$T=123 \mathrm{~K}$

Block, colourless

$0.29 \times 0.17 \times 0.15 \mathrm{~mm}$

$T_{\min }=0.267, T_{\max }=0.267$

13443 measured reflections

1210 independent reflections

1068 reflections with $I>2 \sigma(I)$

$R_{\text {int }}=0.051$

$\theta_{\max }=30.5^{\circ}, \theta_{\min }=3.4^{\circ}$

$h=-9 \rightarrow 9$

$k=-12 \rightarrow 12$

$l=-10 \rightarrow 10$

Hydrogen site location: difference Fourier map

All H-atom parameters refined

$w=1 /\left[\sigma^{2}\left(F_{\mathrm{o}}{ }^{2}\right)+(0.0256 P)^{2}\right]$

where $P=\left(F_{\mathrm{o}}{ }^{2}+2 F_{\mathrm{c}}{ }^{2}\right) / 3$

$(\Delta / \sigma)_{\max }=0.001$

$\Delta \rho_{\max }=0.56 \mathrm{e} \AA^{-3}$

$\Delta \rho_{\min }=-0.86$ e $\AA^{-3}$ 


\section{Special details}

Geometry. All esds (except the esd in the dihedral angle between two 1.s. planes) are estimated using the full covariance matrix. The cell esds are taken into account individually in the estimation of esds in distances, angles and torsion angles; correlations between esds in cell parameters are only used when they are defined by crystal symmetry. An approximate (isotropic) treatment of cell esds is used for estimating esds involving l.s. planes.

Fractional atomic coordinates and isotropic or equivalent isotropic displacement parameters $\left(\AA^{2}\right)$

\begin{tabular}{lllll}
\hline & $x$ & $y$ & $z$ & $U_{\text {iso }}{ }^{*} U_{\text {eq }}$ \\
\hline $\mathrm{Rb} 1$ & $0.24027(2)$ & $0.64932(2)$ & $0.44893(2)$ & $0.01283(6)$ \\
$\mathrm{O} 1$ & $0.6929(2)$ & $0.68315(14)$ & $0.5959(2)$ & $0.0159(2)$ \\
$\mathrm{N} 1$ & $0.7933(2)$ & $0.67488(17)$ & $0.4770(2)$ & $0.0163(3)$ \\
$\mathrm{N} 2$ & $0.8203(2)$ & $0.79640(17)$ & $0.3938(2)$ & $0.0159(3)$ \\
$\mathrm{C} 1$ & $0.7331(3)$ & $0.9368(2)$ & $0.4396(3)$ & $0.0194(3)$ \\
$\mathrm{H} 2$ & $0.583(3)$ & $0.928(2)$ & $0.408(4)$ & $0.027(6)^{*}$ \\
$\mathrm{H} 3$ & $0.753(3)$ & $1.018(2)$ & $0.352(3)$ & $0.016(5)^{*}$ \\
$\mathrm{H} 1$ & $0.812(4)$ & $0.967(3)$ & $0.587(5)$ & $0.049(9)^{*}$ \\
\hline
\end{tabular}

Atomic displacement parameters $\left(\AA^{2}\right)$

\begin{tabular}{lllllll}
\hline & $U^{11}$ & $U^{22}$ & $U^{33}$ & $U^{12}$ & $U^{13}$ & $U^{23}$ \\
\hline $\mathrm{Rb} 1$ & $0.01432(9)$ & $0.01265(9)$ & $0.01354(9)$ & $0.00103(6)$ & $0.00777(6)$ & $0.00118(6)$ \\
$\mathrm{O} 1$ & $0.0194(6)$ & $0.0169(6)$ & $0.0160(6)$ & $-0.0005(4)$ & $0.0120(5)$ & $0.0004(5)$ \\
$\mathrm{N} 1$ & $0.0190(7)$ & $0.0167(8)$ & $0.0158(7)$ & $0.0019(5)$ & $0.0098(6)$ & $0.0009(6)$ \\
$\mathrm{N} 2$ & $0.0204(7)$ & $0.0145(6)$ & $0.0163(7)$ & $0.0029(6)$ & $0.0112(6)$ & $0.0019(6)$ \\
$\mathrm{C} 1$ & $0.0294(9)$ & $0.0126(8)$ & $0.0223(9)$ & $0.0019(7)$ & $0.0167(8)$ & $0.0002(7)$ \\
\hline
\end{tabular}

Geometric parameters $\left(\AA,{ }^{\circ}\right)$

\begin{tabular}{|c|c|c|c|}
\hline $\mathrm{Rb} 1-\mathrm{Rb} 1^{\mathrm{i}}$ & $4.2210(2)$ & $\mathrm{N} 2-\mathrm{Rb}^{\mathrm{v}}$ & $3.0313(14)$ \\
\hline $\mathrm{Rb} 1-\mathrm{Rb} 1^{\mathrm{ii}}$ & $4.2365(3)$ & $\mathrm{N} 2-\mathrm{Rb} 1^{\mathrm{vi}}$ & $3.0761(15)$ \\
\hline $\mathrm{Rb} 1-\mathrm{Rb} 1^{\mathrm{iii}}$ & $5.2757(2)$ & $\mathrm{N} 2-\mathrm{C} 1$ & $1.465(2)$ \\
\hline $\mathrm{Rb} 1-\mathrm{C} 1^{\text {iv }}$ & $3.7471(18)$ & $\mathrm{C} 1-\mathrm{Rb} 1^{\mathrm{i}}$ & $3.7471(18)$ \\
\hline $\mathrm{O} 1-\mathrm{Rb} 1^{\mathrm{i}}$ & $2.8496(12)$ & $\mathrm{C} 1-\mathrm{Rb} 1^{\mathrm{vi}}$ & $3.6538(18)$ \\
\hline $\mathrm{O} 1-\mathrm{Rb} 1^{\mathrm{ii}}$ & $2.9871(12)$ & $\mathrm{C} 1-\mathrm{Rb} 1^{\mathrm{vii}}$ & 3.7031 (19) \\
\hline $\mathrm{O} 1-\mathrm{N} 1$ & $1.3074(19)$ & $\mathrm{C} 1-\mathrm{H} 2$ & $0.97(2)$ \\
\hline $\mathrm{N} 1-\mathrm{Rb} 1^{\mathrm{v}}$ & $3.1656(15)$ & $\mathrm{C} 1-\mathrm{H} 3$ & $1.00(2)$ \\
\hline $\mathrm{N} 1-\mathrm{Rb} 1^{\mathrm{ii}}$ & $2.9173(15)$ & $\mathrm{C} 1-\mathrm{H} 1$ & $1.02(3)$ \\
\hline $\mathrm{N} 1-\mathrm{N} 2$ & $1.274(2)$ & & \\
\hline 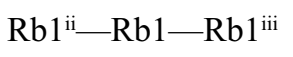 & $113.582(6)$ & $\mathrm{Rb} 1^{\mathrm{vii}}-\mathrm{C} 1-\mathrm{Rb} 1^{\mathrm{i}}$ & $90.16(4)$ \\
\hline 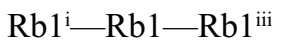 & $85.861(5)$ & $\mathrm{Rb} 1^{\mathrm{vi}}-\mathrm{C} 1-\mathrm{Rb} 1^{\mathrm{i}}$ & $156.42(6)$ \\
\hline 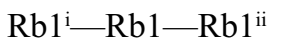 & $77.186(4)$ & $\mathrm{Rb} 1^{\mathrm{vi}}-\mathrm{C} 1-\mathrm{Rb} 1^{\mathrm{vii}}$ & $113.28(5)$ \\
\hline $\mathrm{C} 1^{\mathrm{iv}}-\mathrm{Rb} 1-\mathrm{Rb} 1^{\mathrm{ii}}$ & $73.60(3)$ & 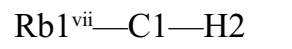 & $94.8(13)$ \\
\hline $\mathrm{C} 1^{\mathrm{iv}}-\mathrm{Rb} 1-\mathrm{Rb} 1^{\mathrm{iii}}$ & $67.80(3)$ & $\mathrm{Rb} 1 \mathrm{i}-\mathrm{C} 1-\mathrm{H} 2$ & $78.4(15)$ \\
\hline $\mathrm{C} 1^{\mathrm{iv}}-\mathrm{Rb} 1-\mathrm{Rb} 1^{\mathrm{i}}$ & $127.36(3)$ & $\mathrm{Rb} 1^{\mathrm{vi}-} \mathrm{C} 1-\mathrm{H} 2$ & $101.1(15)$ \\
\hline 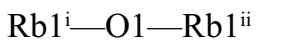 & $129.33(5)$ & $\mathrm{Rb} 1^{\mathrm{vi}}-\mathrm{C} 1-\mathrm{H} 3$ & $58.0(12)$ \\
\hline $\mathrm{N} 1-\mathrm{O} 1-\mathrm{Rb} 1^{\mathrm{ii}}$ & $74.24(8)$ & 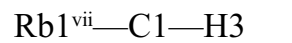 & $55.4(12)$ \\
\hline
\end{tabular}




$\begin{array}{llll}\mathrm{N} 1-\mathrm{O} 1-\mathrm{Rb} 1^{\mathrm{i}} & 136.30(10) & \mathrm{Rb} 1^{\mathrm{i}}-\mathrm{C} 1-\mathrm{H} 3 & 144.9(12) \\ \mathrm{Rb} 1^{\mathrm{ii}}-\mathrm{N} 1-\mathrm{Rb}^{\mathrm{v}} & 95.44(4) & \mathrm{Rb} 1^{\mathrm{vii}}-\mathrm{C} 1-\mathrm{H} 1 & 63.8(16) \\ \mathrm{O} 1-\mathrm{N} 1-\mathrm{Rb} 1^{\mathrm{v}} & 146.43(12) & \mathrm{Rb} 1^{\mathrm{i}}-\mathrm{C} 1-\mathrm{H} 1 & 39.6(15) \\ \mathrm{O} 1-\mathrm{N} 1-\mathrm{Rb} 1^{\mathrm{ii}} & 80.21(8) & \mathrm{Rb} 1^{\mathrm{vi}}-\mathrm{C} 1-\mathrm{H} 1 & 149.8(14) \\ \mathrm{N} 2-\mathrm{N} 1-\mathrm{Rb}^{\mathrm{v}} & 72.28(9) & \mathrm{N} 2-\mathrm{C} 1-\mathrm{Rb} 1^{\mathrm{vii}} & 152.09(11) \\ \mathrm{N} 2-\mathrm{N} 1-\mathrm{Rb} 1^{\mathrm{ii}} & 159.82(11) & \mathrm{N} 2-\mathrm{C} 1-\mathrm{Rb} 1^{\mathrm{i}} & 102.26(10) \\ \mathrm{N} 2-\mathrm{N} 1-\mathrm{O} 1 & 118.93(13) & \mathrm{N} 2-\mathrm{C} 1-\mathrm{Rb} 1^{\mathrm{vi}} & 55.69(8) \\ \mathrm{Rb} 1^{\mathrm{v}}-\mathrm{N} 2-\mathrm{Rb}^{\mathrm{vi}} & 87.43(4) & \mathrm{N} 2-\mathrm{C} 1-\mathrm{H} 2 & 112.1(13) \\ \mathrm{N} 1-\mathrm{N} 2-\mathrm{Rb} 1^{\mathrm{v}} & 84.13(10) & \mathrm{N} 2-\mathrm{C} 1-\mathrm{H} 3 & 106.3(11) \\ \mathrm{N} 1-\mathrm{N} 2-\mathrm{Rb} 1^{\mathrm{vi}} & 128.81(11) & \mathrm{N} 2-\mathrm{C} 1-\mathrm{H} 1 & 11.5(16) \\ \mathrm{N} 1-\mathrm{N} 2-\mathrm{C} 1 & 116.27(13) & \mathrm{H} 2-\mathrm{C} 1-\mathrm{H} 3 & 109(2) \\ \mathrm{C} 1-\mathrm{N} 2-\mathrm{Rb} 1^{\mathrm{v}} & 141.49(11) & \mathrm{H} 2-\mathrm{C} 1-\mathrm{H} 1 & 109.3(19) \\ \mathrm{C} 1-\mathrm{N} 2-\mathrm{Rb} 1^{\mathrm{vi}} & 101.15(10) & \mathrm{H} 3-\mathrm{C} 1-\mathrm{H} 1 & \end{array}$

Symmetry codes: (i) $x+1 / 2,-y+3 / 2, z+1 / 2$; (ii) $-x+1,-y+1,-z+1$; (iii) $-x+1 / 2, y+1 / 2,-z+1 / 2$; (iv) $x-1 / 2,-y+3 / 2, z-1 / 2$; (v) $x+1, y, z$; (vi) $x+1 / 2,-y+3 / 2$, $z-1 / 2$; (vii) $-x+1,-y+2,-z+1$. 